\title{
Creation and Use of a Brettanomyces Aroma Wheel
}

\author{
C.M. Lucy Joseph, ${ }^{1}$ \\ Elizabeth Albino, ${ }^{2}$ \\ and Linda F. Bisson ${ }^{3 *}$
}

Cite this article:

Joseph CML, Albino E and

Bisson LF. 2017. Creation

and use of a Brettanomyces Aroma Wheel. Catalyst 1:12-

20.

${ }^{1}$ Department of Viticulture and Enology, University of California, Davis, One Shields Avenue, Davis, CA 95616; and ${ }^{2}$ Codexis, 200 Penobscot Drive, Redwood City, CA 94063.

*Corresponding author (lfbisson@ucdavis.edu; tel: 530752-1809; fax: 530-752-0382)

Manuscript submitted Apr 2016, revised Aug 2016, accepted Aug 2016

Copyright (c) 2017 by the American Society for Enology and Viticulture. All rights reserved.

doi: $10.5344 /$ catalyst.2016.16003

\section{Summary}

Goals: The ability of the yeast Brettanomyces to produce negative aroma attributes from grape phenolic precursors is well known. However, this yeast synthesizes a broad array of aroma-active compounds in wines, some of which are valued or deemed positive in certain wine styles or matrices. To better understand the spectrum of positive and negative aroma traits associated with the presence of Brettanomyces in wine, we created an aroma wheel to categorize and describe the variety of aroma impacts of this yeast on the basis of analyses of aroma-active compound production from spiked precursor compounds in a model controlled environment.

\section{Key Findings:}

- The Brettanomyces aroma wheel categorizes many of the aroma descriptors associated with wine spoilage by Brettanomyces.

- Terms on the wheel may categorize aroma-active compounds generally associated with microbial infection of both Brettanomyces and lactic acid bacteria (LAB).

- There is no evidence for a Brettanomyces strain that produces only positive characters in wine.

Impact and Significance: A comprehensive Brettanomyces aroma wheel was generated with different strains under controlled product-precursor conditions and tested for broader utility in commercial winemaking. Thirty commercial wines described by wine critics using two or more of the identical or similar terms to those found on the Brettanomyces aroma wheel were obtained from a retail outlet. All wines purchased had evidence of presence of Brettanomyces, $\mathrm{LAB}$, or both. The inner circle, or general categories of the wheel, were used more commonly by critics in describing wines and the specific terms (outer wheel) less generally used as commercial wine descriptors. The terms presented on the aroma wheel can therefore be used to determine if a wine is likely to have sensory characteristics contributed by Brettanomyces or LAB. This appeared to be particularly true for the general categories "savory," "woody," "spicy," "floral," "earthy," "chemical," and "animal." Thus, the wheel can be used to identify wines with a strong, but not necessarily negative, microbial signature.

Key words: Brettanomyces, ethylphenol, lactic acid bacteria, odor-active compound, wine aroma

\section{Overview}

The yeast Brettanomyces was initially found in beer in the early 1900 s by Claussen ${ }^{1}$, but much more research has since been conducted on its role as a spoilage agent in wine. Brettanomyces was identified as the cause of off-characters in South African white wines by van der Walt and van Kerken ${ }^{2}$. Seminal studies by Heresztyn, Chatonnet et al., and Licker et al. ${ }^{3}$ identified some of the primary compounds associated with the Brettanomyces taint and investigated the conditions required to produce them. These volatile phenolic taints are derived from grape precursors. The two most commonly formed compounds, 
4-ethyl phenol (4EP) and 4-ethyl guaiacol (4EG), are described as "animal," "barnyard," or "sweaty horse" or as "medicinal," "BandAid ${ }^{\mathrm{TM}}$," or "smoky." However, more recent work showed that a wider variety of aroma compounds are produced by Brettanomyces from different substrates and different chemical conditions in the wine ${ }^{4}$. Some of these characters would also be generally described as negative, such as "rancid" and "burning tires," whereas others are positive, that is, "floral" and enhanced varietal characteristics.

Anecdotal reports of Brettanomyces found in certain wineries giving only positive aroma characteristics prompted winemakers to express interest in the characterization of these strains and the identification of Brettanomyces strains that could be safely used as inocula. To address this interest, we screened the 99 independent isolates of Brettanomyces that existed in the University of California Davis Department of Viticulture and Enology Wine Yeast and Bacteria Culture Collection to assess the spectrum of end products produced ${ }^{5}$. We conducted these studies in a standard synthetic medium supporting the growth of Brettanomyces with and without the presence of ethanol ${ }^{6}$. The use of a synthetic medium enabled full control of the type and level of precursors used as supplements and facilitated product analysis by gas chromatography (GC). In addition, olfactometry GC was used to identify the specific aroma-active compounds associated with specific compounds produced by the yeast using panelists. These studies were augmented by analyses of Brettanomyces in actual red wines spiked with different compounds. In these analyses, panelists also assessed the aroma-active characters present in these simple growth solutions as well as in the wine studies ${ }^{5}$. In order to facilitate categorization of the aroma descriptors the panelists used, and to distinguish the different strain and substrate effects ${ }^{5}$, we created a Brettanomyces aroma wheel.

During these studies, winemakers sent wines for analysis that contained high levels of volatile phenols as determined by chemical analysis, sometimes an order of magnitude above the published level of detection ${ }^{7}$, but that did not display the common aroma traits associated with these compounds by nose. The wines were sent in hopes that the strains isolated would prove useful as inocula for wine production. The isolated strains were also included in our previous studies to determine if these strains produced masking compounds, either alternative aroma characters made by the yeast or enhanced from the grape.
An alternative explanation, however, was that the matrix of the wine, and not the yeast, was responsible for the masking of these characters. Under defined conditions and in different wine matrices, all of these strains could produce sensorially detectable levels of volatile phenols.

A wide variety of Brettanomyces strains were examined under different substrate and oxygen conditions and a large number of aroma-active compounds were identified $^{5}$ (summarized in Table 1). All B. bruxellensis strains studied could make the compounds 4EP and 4EG, which are considered to be the hallmark of Brettanomyces spoilage under low-oxygen conditions when the two cinnamic acids, coumaric and ferulic acid, are present. However, in the presence of other substrates under other conditions, such as amino acids under higher oxygen, these strains can also make a wide variety of other aroma-active compounds. From our previous studies, the major metabolic compounds produced are often not aroma active, and those that contribute to the aroma are often produced in very low amounts ${ }^{5}$. However, the major sensorially active compounds are often closely related to the major products produced by Brettanomyces ${ }^{5}$.

In the course of this work, we identified 44 compounds either as being major volatile compounds or as compounds that were detectable, using humans as aroma detectors in collaboration with the gas chromatogram and a mass spectral detector (Table 1). Twenty-three (52\%) of those compounds were dependent on the substrate that was in the medium and the strain that was assayed. Ten compounds (23\%) were dependent only on the substrate availability, and another five $(11 \%)$ were produced only by some of the tested strains, regardless of the substrate. Six (14\%) did not depend on either the strain or the substrate used in the test and were produced by Brettanomyces under all conditions $\mathrm{s}^{5}$.

The Brettanomyces aroma wheel created with the panelists during these studies potentially represented a useful practical tool to assist in defining the broader aroma impacts of Brettanomyces in commercial wines. We assessed the validity of this aroma wheel for the analysis of commercial wines in two ways: (1) by purchasing wines that had been described by wine critics in terms consistent with those found on the Brettanomyces aroma wheel and evaluating those wines for the presence of Brettanomyces, and (2) by using the wheel in an extension program with more than 100 winemakers in attendance, with tastings of wines spiked with various substrates and strains of Brettanomyces 
and obtaining assessments of aroma characters from the audience. This paper presents the aroma wheel and the validation thereof, using marketplace wines selected on the basis of commercial descriptions matching multiple terms on the wheel.

\section{Major Observations and Interpretations}

The completed aroma wheel is presented in Figure 1. We determined these descriptive terms in a synthetic

Table 1 Chemical compounds produced by different strains of Brettanomyces bruxellensis and the aromas they produce.

\begin{tabular}{|c|c|c|c|c|c|}
\hline $\begin{array}{l}\text { Chemical compound } \\
\text { and CAS number }\end{array}$ & $\begin{array}{c}\text { Type of } \\
\text { compound }\end{array}$ & $\begin{array}{l}\text { Substrate } \\
\text { dependent }\end{array}$ & $\begin{array}{c}\text { Strain } \\
\text { dependent }\end{array}$ & $\begin{array}{c}\text { Major } \\
\text { product }\end{array}$ & Aroma* $^{*}$ \\
\hline 2-Ethyl-1-hexanol 104-76-7 & Ester & Yes & No & Yes & Citrus, floral \\
\hline 2-Methyl-1-butanol 137-32-6 & Alcohol & Yes & No & Yes & Canned fruit, plastic \\
\hline 3-Methyl-1-butanol (isoamyl) 123-51-3 & 3 Alcohol & No & No & Yes & Banana, whiskey, chemical \\
\hline 4-Ethyl guaiacol 2785-89-9 & Phenolic & Yes & No & Yes & Smoky, clove, spice, phenolic \\
\hline 4-Ethyl phenol 123-07-9 & Phenolic & Yes & No & Yes & Phenolic, creosote, band-aid \\
\hline Ethyl 2-methyl butyrate 7452-79-1 & Ester & Yes & Yes & Yes & Mint, citrus, green apple \\
\hline Phenethyl alcohol 60-12-8 & Alcohol, ester & No & No & Yes & Floral, rose \\
\hline 1-Decanol 112-30-1 & Alcohol & Yes & Yes & Yes & Waxy, floral, orange \\
\hline 1-Octanol 111-87-5 & Alcohol & Yes & Yes & Yes & Citrus, waxy, aldehydic, floral \\
\hline 2-Methyl butyric acid 116-53-0 & Fatty acid, ester & Yes & Yes & Yes & Blue cheese, rancid \\
\hline 2-Nonanone 821-55-6 & Ketone & No & No & Yes & Fruity, soapy, herbaceous \\
\hline $\begin{array}{l}\text { 3-Methyl butyric acid (isovaleric) } \\
\text { 503-74-2 }\end{array}$ & Fatty acid, ester & Yes & Yes & Yes & Sweaty feet, cheese \\
\hline Acetic acid 64-19-7 & Organic acid & No & Yes & Yes & Vinegar, sour \\
\hline$\beta$-Farnesene 18794-84-8 & Terpene & Yes & Yes & Yes & Woody \\
\hline Butanol 71-36-3 & Alcohol & Yes & No & Yes & Alcohol \\
\hline Decanoic acid 334-48-5 & Fatty acid & Yes & No & Yes & Rancid, sour, fatty \\
\hline Ethyl acetate $141-78-6$ & Ester & No & Yes & Yes & Pear, apple, nail polish remover \\
\hline Ethyl decanoate 110-38-3 & Ester & Yes & Yes & Yes & Fruity, apple, waxy \\
\hline Ethyl dodecanoate 106-33-2 & Ester & Yes & Yes & Yes & Soapy, rum, clean \\
\hline Ethyl isobutyrate $97-62-1$ & Ester & Yes & No & Yes & Fruity, rum \\
\hline Ethyl octanoate 106-32-1 & Ester & No & No & Yes & Fruity, pineapple, apricot \\
\hline Ethyl tetradecanoate $124-06-1$ & Ester & Yes & No & Yes & Waxy, violet \\
\hline Isobutyric acid 79-31-2 & Fatty acid & Yes & Yes & Yes & Rancid, cheese \\
\hline Octanoic acid 124-07-2 & Fatty acid & Yes & No & Yes & Rancid, cheesy \\
\hline Pentanoic acid 109-52-4 & Fatty acid & No & No & Yes & Putrid, rancid, sweat, cheese, \\
\hline Phenethyl acetate 103-45-7 & Ester & Yes & Yes & Yes & Floral, rose, honey \\
\hline Phenethyl propionate 103-52-6 & Ester & No & Yes & Yes & Musty, floral, yeasty \\
\hline Phenylacetaldehyde 122-78-1 & Aldehyde & No & Yes & Yes & Floral, honey \\
\hline 2-Methoxy-4-vinylphenol 7786-61-0 & Phenolic & Yes & Yes & No & Woody, cedar, roasted nuts \\
\hline $\begin{array}{l}\text { 4-Methoxyphenethyl methanol } \\
\text { 105-13-5 }\end{array}$ & Alcohol, ester & Yes & Yes & No & Floral, balsamic, fruit, anise \\
\hline Amyl-octanoate $638-25-5$ & Fatty acid & Yes & Yes & No & Wine, elderflower, orris \\
\hline Bisabolene 495-62-5 & Terpene & Yes & Yes & No & $\begin{array}{l}\text { Woody, citrus, tropical fruit, green } \\
\text { banana }\end{array}$ \\
\hline Butyric acid 107-92-6 & Fatty acid & Yes & Yes & No & Fruity, cheesy, acetic \\
\hline Ethyl butyrate 105-54-4 & Ester & Yes & Yes & No & Tutti-frutti, pineapple, cognac \\
\hline Ethyl isovalerate $108-64-5$ & Ester & Yes & No & No & Fruity, esters, sharp, pineapple \\
\hline Ethyl valerate $539-82-2$ & Ester & Yes & Yes & No & Tropical fruit, strawberry, pineapple \\
\hline Heptanoic acid 11-14-08 & Fatty acid & Yes & Yes & No & Fatty, animal \\
\hline Isoamyl alcohol 125-51-3 & Alcohol & No & No & No & Fruity, banana, whiskey \\
\hline Nonanal 124-19-6 & Aldehyde & Yes & Yes & No & Citrus, waxy, melon, aldehydic \\
\hline Ocimene 502-99-8 & Terpene & Yes & Yes & No & Fruity, floral, wet cloth \\
\hline Octyl butyrate $110-39-4$ & Fatty acid, ester & Yes & Yes & No & Fruity, oily, fresh or green, earthy \\
\hline Pentyl formate $638-49-3$ & Ester & Yes & Yes & No & Fruity, unripe banana, earthy \\
\hline Phenethyl formate 104-62-1 & Ester & Yes & Yes & No & Floral, green, watercress, hyacinth \\
\hline Undecanoic acid 112-37-8 & Fatty acid & No & Yes & No & Creamy, fatty coconut \\
\hline
\end{tabular}

*Descriptors derived from The Good Scents Company website: http://www.thegoodscentscompany.com/. 
environment to define specific product-precursor relationships and confirm production of odor-active compounds by pure cultures of Brettanomyces. During discussions with panelists evaluating the synthetic samples, we organized the outer wheel descriptors into the inner wheel broader categories. For example, the smoky character often associated with Brettanomyces presence in wine was split into two terms-a smoked meat character under "savory" and a burned beans character under "rotten and putrid," but the smoky character was not considered "spicy." Winematrix effects will determine how these characters are perceived in wines, often modulating the more extreme characteristics. The "dairy" characteristics were quite strong in the pure-culture synthetic media for multiple strains of Brettanomyces, but are rarely noted for Brettanomyces infection in commercial wineproduction conditions. The thresholds of detection in synthetic media will obviously differ from those in wines, and detection of the same level of a given compound will vary across different wines. These descriptors may be used as a guide for the types of descriptive terms that indicate microbial impact on wine aroma.

To test the validity of the Brettanomyces aroma wheel, we took advantage of the wine descriptions posted by an online wine retailer, K\&L Wine Merchants (klwines.com). This site publishes wine descriptions and reviews from a number of sources in a searchable format for wines available for purchase. We searched using specific terms on the wheel and identified 30 wines that were described with multiple terms on the wheel as candidates for assessing the presence of Brettanomyces. We also noted whether these wines were described with terms related to those on the aroma wheel. None of these wines were described using the "fermentation," dairy, rotten/putrid, or "veggie" descriptors associated with Brettanomyces spoilage, and none of these aroma traits were detected in our own sensory analyses of these wines. We were more interested in determining whether other Brettanomyces-associated traits in our research would signal the presence of this yeast in commercial wines. We also noted if wines were described as having been filtered or had any other pertinent processing information (i.e., biodynamic, organic, no added sulfites), but the processing of the selected wines was variable, and none of the reported winemaking procedures was strongly correlated with terms on the wheel. Given what is known about the widespread infections of wine with Brettanomyces, this observation was not surprising. In some cases, we also selected common terms used by wine critics, such as "Asian spice," as this descriptor seemed a hybrid of our tamarind and savory terms when the panel evaluated these wines. We then purchased the 30 wines from a retailer to test for the presence of Brettanomyces (Table 2).

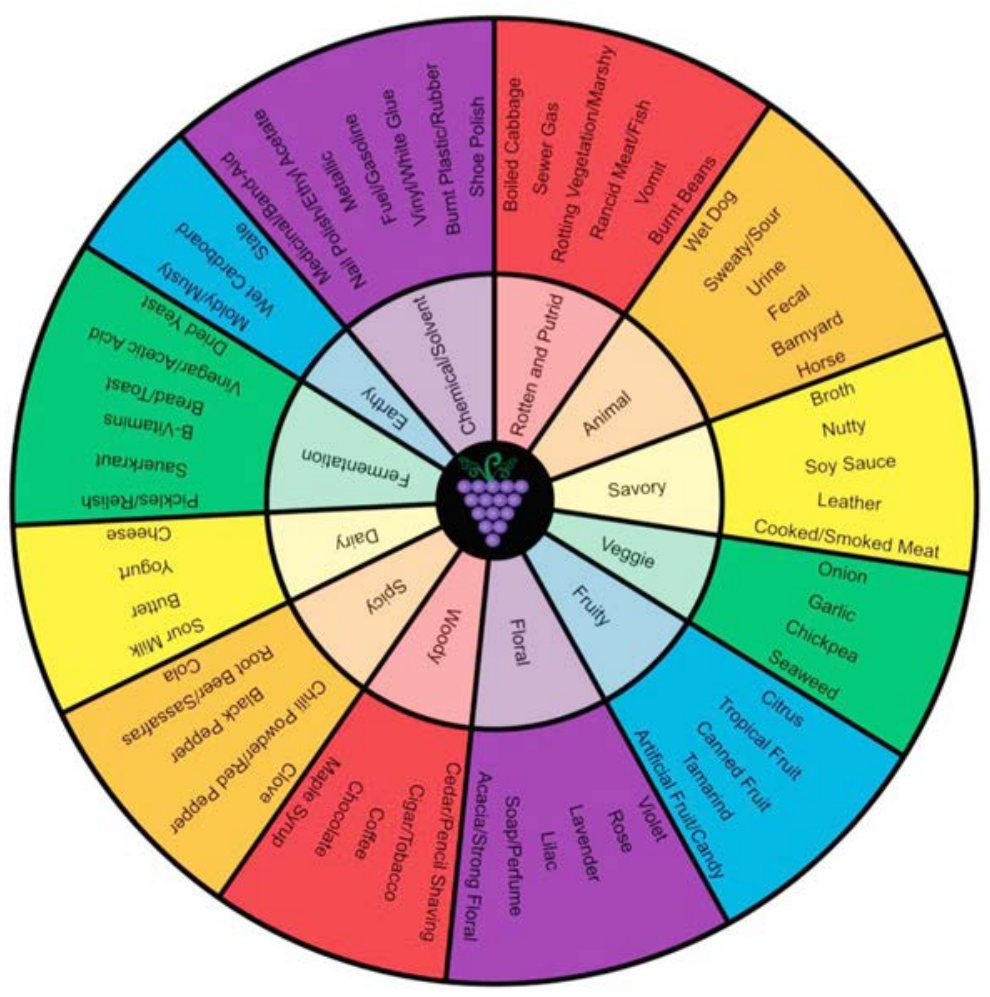

Figure 1 The completed aroma wheel using descriptors for Brettanomyces in synthetic wine and determined by a trained panel of judges. Twelve general categories are broken down to between three and seven more specific terms. The reverse of the wheel (not shown) shows photomicrographs of $B$. bruxellensis and summarizes some of the information given here. 
We used four different methods to determine the presence of Brettanomyces in these wines: (1) microscopic examination, (2) plating on selective media for live organisms, (3) quantitative or real-time polymerase chain reaction (qPCR) to check for Brettanomyces DNA, and (4) chemical analysis to detect the signature compounds 4EP and 4EG produced by Brettanomyces. The initial microscopic and plating examination on Wallerstein Laboratory Differential nutrient medium with cycloheximide (WLD) for Brettanomyces and de Man, Rogosa, and Sharpe medium (MRS) with nystatin for bacteria ${ }^{8}$ revealed that 21 of the wines had viable LAB, and another six had viable Brettanomyces. Of those 27, one (number 14) eventually proved to have both viable Brettanomyces and LAB. A number of these wines, that is, those that had not initially yielded microbial isolates, were also tested with qPCR for Brettanomyces and with HPLC for 4EG and 4EP. The qPCR and HPLC analyses were performed by the analytical wine lab at Treasury Wine Estates in Napa,

\begin{tabular}{|c|c|c|c|c|c|c|c|c|c|}
\hline $\begin{array}{l}\text { Sample } \\
\text { No. }\end{array}$ & Vintage & Variety & $\begin{array}{l}\text { Plating/ } \\
\text { microscopy }\end{array}$ & $\begin{array}{l}\text { Brett qPCR } \\
\text { cells } / \mathrm{mL}\end{array}$ & $\begin{array}{c}4 E P^{a} \\
\mathrm{ng} / \mathrm{mL}\end{array}$ & $\begin{array}{r}4 \mathrm{EG}^{\mathrm{a}} \\
\mathrm{ng} / \mathrm{mL}\end{array}$ & $\begin{array}{l}\text { Microbes }^{a} \\
\text { present }\end{array}$ & Descriptors & $\begin{array}{l}\text { Descriptor } \\
\text { categories }\end{array}$ \\
\hline 1 & 1996 & $\begin{array}{l}\text { Red Bordeaux } \\
\text { blend, France }\end{array}$ & Pediococcus & $<10$ & 11.8 & 6.5 & LAB & Smoke and earth & Earthy, savory \\
\hline 2 & 2004 & $\begin{array}{l}\text { Red Bordeaux } \\
\text { blend, France }\end{array}$ & $\begin{array}{l}\text { Lactobacillus, } \\
\text { Pediococcus, }\end{array}$ & 6900 & 13.7 & 6.9 & LAB, Brett & $\begin{array}{l}\text { Cedar, tobacco, } \\
\text { underbrush, smoke/ } \\
\text { burning embers, toast, } \\
\text { cigar smoke, asian } \\
\text { spices }\end{array}$ & $\begin{array}{l}\text { Savory, spicy, } \\
\text { woody }\end{array}$ \\
\hline 3 & 2005 & $\begin{array}{l}\text { Red, Rioja, } \\
\text { Spain }\end{array}$ & $N G^{b}$ & 1300 & 12.5 & 7.0 & Brett & $\begin{array}{l}\text { Umami, soy sauce, } \\
\text { minerality }\end{array}$ & Savory, earthy \\
\hline 4 & 2006 & $\begin{array}{l}\text { Sangiovese } \\
\text { Grosso, Italy }\end{array}$ & Lactobacillus & 2200 & 13.6 & 7.8 & LAB, Brett & $\begin{array}{l}\text { Cooked meat, tar, } \\
\text { smoke, new leather, } \\
\text { forest floor, and root } \\
\text { beer }\end{array}$ & $\begin{array}{l}\text { Savory, } \\
\text { chemical, spicy, } \\
\text { earthy }\end{array}$ \\
\hline 5 & 2007 & $\begin{array}{l}\text { Sangiovese, } \\
\text { Italy }\end{array}$ & $\begin{array}{l}\text { Pediococcus, } \\
\text { Lactobacillus }\end{array}$ & 1600 & 12.5 & 7.5 & LAB, Brett & $\begin{array}{l}\text { Musky, black truffle, } \\
\text { roses, spice, tobacco, } \\
\text { ginger, cola, leather, } \\
\text { and game }\end{array}$ & $\begin{array}{l}\text { Earthy, floral, } \\
\text { spicy, woody, } \\
\text { savory, animal }\end{array}$ \\
\hline 6 & 2007 & $\begin{array}{l}\text { Red Bordeaux } \\
\text { blend, Australia }\end{array}$ & Brettanomyces & 13,000 & 12.9 & 7.2 & Brett & $\begin{array}{l}\text { Spice box, violets, } \\
\text { pencil lead, leather, } \\
\text { warm earth, and sweet } \\
\text { tobacco }\end{array}$ & $\begin{array}{l}\text { Spicy, floral, } \\
\text { woody, savory, } \\
\text { earthy, }\end{array}$ \\
\hline 7 & 2008 & $\begin{array}{l}\text { Tempranillo, } \\
\text { Spain }\end{array}$ & Lactobacillus & 1600 & 12.7 & 7.3 & LAB, Brett & $\begin{array}{l}\text { Beef jerky, meaty, } \\
\text { spicy, savory }\end{array}$ & Savory, spicy \\
\hline 8 & 2008 & $\begin{array}{l}\text { Red Bordeaux } \\
\text { blend, France }\end{array}$ & Lactobacillus & $<10$ & 10.8 & 6.8 & LAB & $\begin{array}{l}\text { Smoke, pencil } \\
\text { shavings }\end{array}$ & Savory, woody \\
\hline 9 & 2009 & $\begin{array}{l}\text { Rhone blend, } \\
\text { France }\end{array}$ & Lactobacillus & $<10$ & 10.5 & 7.0 & LAB & $\begin{array}{l}\text { Mineral, spicy, black } \\
\text { tea, roasted mesquite, } \\
\text { graphite, charcoal, } \\
\text { truffle, leather, and } \\
\text { earth }\end{array}$ & $\begin{array}{l}\text { Earthy, spicy, } \\
\text { savory }\end{array}$ \\
\hline 10 & 2009 & $\begin{array}{l}\text { Cabernet } \\
\text { Sauvignon, } \\
\text { France }\end{array}$ & $\begin{array}{l}\text { Pediococcus, } \\
\text { Lactobacillus }\end{array}$ & & & & LAB & $\begin{array}{l}\text { Scorched earth/burning } \\
\text { embers/charcoal, } \\
\text { pencil shavings, rose, } \\
\text { gardenia and violet, } \\
\text { oriental spice, flinty } \\
\text { mineral, graphite, } \\
\text { tobacco, and crushed } \\
\text { stone }\end{array}$ & $\begin{array}{l}\text { Savory, earthy, } \\
\text { woody, floral, } \\
\text { spicy }\end{array}$ \\
\hline 11 & 2009 & $\begin{array}{l}\text { Pinot noir, } \\
\text { California }\end{array}$ & Pediococcus & & & & LAB & $\begin{array}{l}\text { Asian spices, meat } \\
\text { stock, soy, mineral, } \\
\text { umami, incense, and } \\
\text { cola }\end{array}$ & $\begin{array}{l}\text { Spicy, savory, } \\
\text { earthy }\end{array}$ \\
\hline 12 & 2009 & $\begin{array}{l}\text { Charbono, } \\
\text { California }\end{array}$ & Pediococcus & & & & LAB & Root beer, minerality & Spicy, earthy, \\
\hline 13 & 2009 & $\begin{array}{l}\text { Grenache-Mataro- } \\
\text { Shiraz blend, } \\
\text { Australia }\end{array}$ & Lactobacillus & & & & LAB & $\begin{array}{l}\text { Spicecake, funk, and } \\
\text { earth plus a little tar }\end{array}$ & $\begin{array}{l}\text { Spicy, earthy, } \\
\text { chemical }\end{array}$ \\
\hline 14 & 2010 & $\begin{array}{c}\text { Grenache-Syrah- } \\
\text { Mourvedre blend, } \\
\text { France }\end{array}$ & $\begin{array}{l}\text { Lactobacillus, } \\
\text { Brettanomyces }\end{array}$ & $<10$ & 1529 & 450 & LAB, Brett & $\begin{array}{l}\text { Spices, smoked } \\
\text { meat, acacia flowers, } \\
\text { graphite, scorched } \\
\text { earth }\end{array}$ & $\begin{array}{l}\text { Spicy, savory, } \\
\text { floral, earthy }\end{array}$ \\
\hline
\end{tabular}


CA. The results of these analyses indicated that three additional wines also had Brettanomyces. One of the wines (number 18) had elevated 4EP and 4EG levels, indicating probable Brettanomyces contamination at some point. The other two (numbers 3 and 21) had Brettanomyces DNA as determined by qPCR. In summary, $57 \%$ of the wines had active LAB contamination, and $27 \%$ showed evidence of Brettanomyces contamination. Some of the wines (16\%) showed evidence of both Brettanomyces and LAB. In other words, $100 \%$ of the wines chosen using the descriptors from the Brettanomyces aroma wheel tested positive for microbial spoilage organisms.
Although these terms were developed using pure cultures of Brettanomyces, many of the wines showed evidence of LAB but not of Brettanomyces. This unexpected result may mean that both of these classes of organisms can produce the same aroma compounds from amino acid precursors as has previously been reported for the mousy trait derived from lysine ${ }^{9}$. This is also consistent with our observation of the dairy traits found with Brettanomyces. However, these were commercial wines, and we cannot rule out the possibility that Brettanomyces was present at some point during the lifespan of the wines that tested negative for this yeast in our study.

\begin{tabular}{|c|c|c|c|c|c|c|c|c|c|}
\hline $\begin{array}{l}\text { Sample } \\
\text { No. }\end{array}$ & Vintage & Variety & $\begin{array}{c}\text { Plating/ } \\
\text { microscopy }\end{array}$ & $\begin{array}{l}\text { Brett qPCR } \\
\text { cells/mL }\end{array}$ & $\begin{array}{l}\text { 4EPa } \\
\mathrm{ng} / \mathrm{mL}\end{array}$ & $\begin{array}{l}4 \mathrm{EG}^{\mathrm{a}} \\
\mathrm{ng} / \mathrm{mL}\end{array}$ & $\begin{array}{l}\text { Microbes }^{a} \\
\text { present }\end{array}$ & Descriptors & $\begin{array}{l}\text { Descriptor } \\
\text { categories }\end{array}$ \\
\hline 15 & 2011 & Syrah, France & $\begin{array}{l}\text { Lactobacillus, } \\
\text { Pediococcus }\end{array}$ & & & & LAB & $\begin{array}{l}\text { Smoky, meatiness, } \\
\text { singed vanilla, } \\
\text { tobacco, violet, and } \\
\text { cola }\end{array}$ & $\begin{array}{l}\text { Savory, woody, } \\
\text { spicy, floral }\end{array}$ \\
\hline 16 & 2003 & Syrah, California & Pediococcus & & & & LAB & $\begin{array}{l}\text { Smoked meat, loamy } \\
\text { soil, violets, lavender }\end{array}$ & $\begin{array}{l}\text { Savory, floral, } \\
\text { earthy }\end{array}$ \\
\hline 17 & 2010 & $\begin{array}{l}\text { Cabernet franc, } \\
\text { France }\end{array}$ & Brettanomyces & 7 & 342 & 96 & Brett & Spicy, savory herbs & Spicy, savory \\
\hline 18 & 2009 & $\begin{array}{l}\text { Red Bordeaux } \\
\text { blend, France }\end{array}$ & $N G$ & $<10$ & 335 & 77 & Brett & $\begin{array}{l}\text { Mineral, cedar, violets, } \\
\text { and lavender }\end{array}$ & $\begin{array}{l}\text { Earthy, floral } \\
\text { woody }\end{array}$ \\
\hline 19 & 2007 & $\begin{array}{l}\text { Pinot noir, } \\
\text { California }\end{array}$ & Pediococcus & $<10$ & 9 & 6 & LAB & $\begin{array}{l}\text { Funky/wild, game, } \\
\text { earth, forest floor, } \\
\text { loam, truffles, spice, } \\
\text { graphite }\end{array}$ & $\begin{array}{l}\text { Animal, earthy, } \\
\text { spicy }\end{array}$ \\
\hline 20 & 1999 & $\begin{array}{l}\text { Pinot noir, } \\
\text { California }\end{array}$ & Lactobacillus & & & & LAB & $\begin{array}{l}\text { Umami, violets, forest } \\
\text { floor, chinese five } \\
\text { spice, and mushroom }\end{array}$ & $\begin{array}{l}\text { Savory, earthy, } \\
\text { floral, spicy }\end{array}$ \\
\hline 21 & 2001 & $\begin{array}{l}\text { Tempranillo- } \\
\text { Granache blend, } \\
\text { Spain }\end{array}$ & $N G$ & 1200 & 12.4 & 7.2 & Brett & $\begin{array}{l}\text { Singed plum, balsamic, } \\
\text { sandalwood, potpourri }\end{array}$ & $\begin{array}{l}\text { Savory, woody, } \\
\text { spicy, floral }\end{array}$ \\
\hline 22 & 2004 & $\begin{array}{l}\text { Tempranillo, } \\
\text { Spain }\end{array}$ & Lactobacillus & & & & LAB & $\begin{array}{l}\text { Leather, cinnamon, } \\
\text { ox-blood, smoked meat }\end{array}$ & $\begin{array}{l}\text { Savory, spicy, } \\
\text { animal }\end{array}$ \\
\hline 23 & 2007 & $\begin{array}{l}\text { Cabernet franc, } \\
\text { New Zealand }\end{array}$ & Brettanomyces & & & & Brett & $\begin{array}{l}\text { Cooked meats, wild } \\
\text { game, violets, herbs, } \\
\text { spice, and mineral }\end{array}$ & $\begin{array}{l}\text { Savory, animal, } \\
\text { spicy, earthy, } \\
\text { floral }\end{array}$ \\
\hline 24 & 2009 & $\begin{array}{l}\text { Red Bordeaux } \\
\text { blend, France }\end{array}$ & Brettanomyces & $<10$ & 768 & 124 & Brett & $\begin{array}{l}\text { Black tea, earth, } \\
\text { mocha, spice, tobacco, } \\
\text { and mineral }\end{array}$ & $\begin{array}{l}\text { Earthy, woody, } \\
\text { spicy }\end{array}$ \\
\hline 25 & 2009 & $\begin{array}{l}\text { Mourvedre-Syrah- } \\
\text { Grenache blend, } \\
\text { California }\end{array}$ & Lactobacillus & & & & LAB & $\begin{array}{l}\text { Exotic asian spices, } \\
\text { sandalwood, crushed } \\
\text { rock, mineral, and } \\
\text { cedar }\end{array}$ & $\begin{array}{l}\text { Spicy, woody, } \\
\text { earthy }\end{array}$ \\
\hline 26 & 2009 & Syrah, France & Lactobacillus & & & & LAB & Mineral, smoky, spice & $\begin{array}{l}\text { Earthy, savory, } \\
\text { spicy }\end{array}$ \\
\hline 27 & 2009 & $\begin{array}{l}\text { Cabernet franc, } \\
\text { France }\end{array}$ & Brettanomyces & 11,000 & 869 & 313 & Brett & Gravel and sand & Earthy \\
\hline 28 & 2010 & Garnacha, Spain & Lactobacillus & & & & LAB & $\begin{array}{l}\text { Roasted herbs, spice, } \\
\text { and woodsmoke }\end{array}$ & $\begin{array}{l}\text { Savory, spicy, } \\
\text { woody }\end{array}$ \\
\hline 29 & 2010 & $\begin{array}{l}\text { Mourvedre- } \\
\text { Grenache Rhone } \\
\text { blend, France }\end{array}$ & Lactobacillus & & & & LAB & $\begin{array}{l}\text { Rose, lavender, asian } \\
\text { spices, forest floor, } \\
\text { and truffle, tarry }\end{array}$ & $\begin{array}{l}\text { Floral, spicy, } \\
\text { earthy, chemical }\end{array}$ \\
\hline 30 & 2010 & $\begin{array}{l}\text { Merlot-Cabernet } \\
\text { Sauvignon blend }\end{array}$ & Pediococcus & & & & LAB & $\begin{array}{l}\text { Earth, tobacco, and } \\
\text { roasted cedar }\end{array}$ & $\begin{array}{l}\text { Earthy, woody, } \\
\text { savory }\end{array}$ \\
\hline
\end{tabular}

a4EP, 4-ethyl phenol; 4EG, 4-ethyl guaiacol; LAB, lactic acid bacteria; Brett, Brettanomyces.

${ }^{b} N G=$ No growth .

Blank spaces indicate not determined. 


\section{Broader Impact}

We analyzed these data to look for correlations between the presence of LAB or Brettanomyces and the presence of general descriptor categories (Figure $2)$. This analysis was less than ideal since the backgrounds for these wines differed in each case. The variability in microbial population was not the only difference among the wines. Moreover, the absence of a particular class of organisms does not necessarily mean that members of that class were never present during the life of the wine and merely indicates that they cannot be detected in the final commercial product. Another issue with any analysis is the number of samples in each category: 17 wines had LAB alone, eight had Brettanomyces alone, and five had both Brettanomyces and LAB. Despite these issues, we could see some general trends in the data.

The categories spicy, savory, and earthy accounted for most of the classes of descriptors used for the wines. The woody and floral categories were used less commonly, and only rarely were the animal and "chemical" classifications found. There were no descriptors in the fermentation, dairy, rotten/putrid, veggie, or "fruity" categories. Some of this may reflect the commercially unacceptable nature of wines with these aroma characteristics, as would be the case for rotten/putrid, whereas other aroma-active characters may have been masked in the wine. The absence of fruity may be more a result of the selection procedure we used, as those descriptors were too commonly used to be valuable as selection criteria. The division of the aroma categories by type of microbial contamination typically followed the number of wines with each contamination profile: LAB (57\%), Brettanomyces (27\%), or both (16\%).

Half of the wines with Brettanomyces and $40 \%$ of the wines with both LAB and Brettanomyces had floral descriptors, and only one-third of the wines with LAB had only floral descriptors (Figure 2). A similar trend was seen for the woody descriptor. High numbers of wines with the savory and spicy descriptors had either Brettanomyces or LAB (63 to 77\%, respectively), but $100 \%$ of the wines with both LAB and Brettanomyces had these two types of descriptors. The earthy descriptor was also highly prevalent in all the wines; it was found in $75 \%$ of wines with Brettanomyces, $77 \%$ of wines with LAB, and $60 \%$ of wines with both.

There are a few specific descriptors that seem to occur exclusively with LAB, but none that occurred exclusively with Brettanomyces. One descriptor that stood out for LAB was Asian spice, which also includes Oriental spice and Chinese five spice. Overall, $35 \%$ of the wines with LAB were described as having that character. A few other descriptors occurred less often, but still were exclusively associated with wines that had LAB aromas, such as graphite, truffle, and tar. The low number of samples and the variability in processing prevent us from drawing any definitive conclusions about the specific descriptors and their metabolic origins among the community of microbes present. Further analysis of a greater number of wines could yield more specific descriptors from the outer wheel that are associated with specific types of microbial contamination.

The Brettanomyces aroma wheel was generated as a consequence of multiple analyses of the growth of this yeast in a synthetic matrix with and without supplementation with specific precursor compounds. Brettanomyces can generate an array of aroma compounds in addition to the well-known aromatic phenol derivatives. The wheel was developed in consultation with two panels and was validated by selecting a set of commercial wines that were described using

Percentage of Aroma Category for Each Contaminant

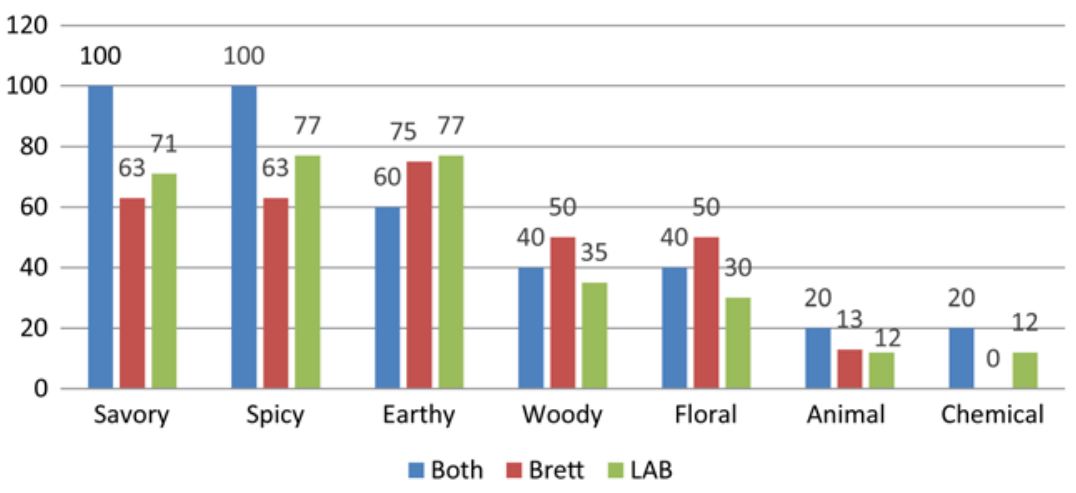

Figure 2 The percentage of the wines with a given microbial contaminant (Brettanomyces, lactic acid bacteria [LAB], or both) that were described as having a specific aroma characteristic. For example, $100 \%$ of wines with both Brettanomyces and LAB and $77 \%$ of wines with $\mathrm{LAB}$ alone were described as spicy. The information was compiled from Table 2. 
multiple terms on the wheel. Although the presence of Brettanomyces was confirmed in only $43 \%$ of these wines, the remainder contained LAB, Lactobacillus, and Pediococcus, suggesting that LAB can produce similar compounds in wine or, alternatively, that Brettanomyces had been present in these wines at some point but was no longer detectable at the time of the evaluation. The Brettanomyces aroma wheel represents a useful tool for assessing the contribution of microbial aromaactive compounds to the aroma profile of wines.

\section{Experimental Design}

The work presented here builds on a series of previously published studies that generated an array of terms for the description of synthetic media inoculated with different strains of Brettanomyces with and without supplementation with specific precursor compounds ${ }^{5}$. Those publications contain more detailed descriptions of the sensory analyses conducted, and we summarize them here. A set of 99 Brettanomyces strains were evaluated for aroma characteristics in minimal media, either unsupplemented or supplemented with phenylalanine, tyrosine, or tryptophan. Aroma terms for the 99 strains were aggregated, for a total of 2646 observations and 90 unique terms. Judges met as a group afterward to clarify terminology in order to aid in descriptor classification, i.e., "coconut oil" referred to a rancid oil smell, and should be categorized as "rotten/ putrid" rather than "fruity" or "spicy." Panel discussions were used to group terms into 1 of 13 classes of related descriptors. Panelists agreed on the overall groupings of their terms, and judges were encouraged to use their own terms. There was no communication among the panelists during the initial sample analyses and no effort was made to force agreement among the individual panelists in the terms used. All samples received randomized three digit codes as assigned by a random-number generator ( $w w w$ w.randomizer.org). Strains were presented in groups of three or four (i.e., as 12 to 16 samples), in a randomized order for each set. Five judges were recruited for the study, all of whom had previous experience with Brettanomyces aroma evaluation. Judges were asked to provide a list of descriptors for each sample; to rate the aroma profile present as "positive," "negative," or "a mix of positive and negative;" and to apply an overall intensity rating on a scale of one through nine.

Descriptors that were used by more than a single panelist during the analyses of both synthetic and actual wine samples were compiled for consideration in the final wheel for discussions with the panelists. The groupings were changed from 13 to 12 by the inclusion of the "acetic" term of the first panel under "fermentation." More general descriptors for certain aroma characters were also chosen after the discussion. For example, fuel and gasoline were combined into a single term, and "boiled cabbage" was used for "pot stickers," "kimchee," and "stuffed cabbage." The more specific terms (on the outer wheel) were then categorized into more general terms, such as fruity, floral, rotten and putrid, or animal (on the inner wheel).

The first version of the wheel was tested during an extension program focused on identifying the impacts of Brettanomyces in wine. Commercial wines deliberately made with Brettanomyces were evaluated during this program as a series of Merlot wines that we had produced through inoculation with different Brettanomyces strains. Attendees were asked to evaluate the wines, write descriptors, and then consult the Brettanomyces aroma wheel to determine if the descriptors were present. Some terms were modified slightly, but no new terms were suggested, and none were suggested for removal.

Acknowledgments: The authors thank Treasury Wine Estates, Tom Collins, and Josh Miles for qPCR and HPLC wine analysis.

\section{References and Footnotes}

1. Claussen NH. 1904. On a method for the application of Hansen's pure yeast system in the manufacturing of wellconditioned English stock beers. J Inst Brew 10:308-331.

2. Van Der Walt JP and Van Kerken AE. 1958. The wine yeasts of the cape. Part I.-A taxonomical survey of the yeasts causing turbidity on South African table wines. Ant Leeuwenhoek 24:239-252.

3. Heresztyn T. 1986. Metabolism of volatile phenolic compounds from hydroxycinnamic acids by Brettanomyces yeast. Arch Microbiol 146:96-98.

Chatonnet P, Dubourdieu D and Boidron JN. 1995. The influence of Brettanomyces/Dekkera sp. yeasts and lactic acid bacteria on the ethylphenol content of red wine. Am J Enol Vitic 46:463-468.

Licker JL, Acree TE and Henick-Kling T. 1998. What is "Brett" (Brettanomyces) flavor?: A preliminary investigation. In Chemistry of Wine Flavor, ACS Symposium Series, vol. 714. Waterhouse AL and Ebeler SE (eds), pp. 96-115. American Chemical Society, Washington DC.

4. Hesford F, Schneider K, Porret N and Gafner J. 2004. Identification and analysis of 4-ethyl catechol in wines tainted by Brettanomyces off-flavor. Am J Enol Vitic 55:304A.

Culleré L, Escudero A, Chaco J and Ferreira V. 2004. Gas chromatography -olfactometry and chemical quantitative study of the aroma of six premium quality Spanish aged red wines. J Agric Food Chem 52:1653-1660. 
Renouf V, Falcou M, Miot-Sertier C, Perello MC, de Revel G and Lonvaud-Funel A. 2006. Interactions between Brettanomyces bruxellensis and other yeast species during the initial stages of winemaking. J Appl Microbiol 100:1208-1219.

Hernández-Orte $\mathrm{P}$, Cersosimo $\mathrm{M}$, Loscos $\mathrm{N}$, Cacho J, Garcia-Moruno E and Ferreira V. 2008. The development of varietal aroma from non-floral grapes by yeasts of different genera. Food Chem 107:1064-1077.

Romano A, Perello MC, Lonvaud-Funel A, Sicard G, and de Revel G. 2009. Sensory and analytical re-evaluation of "Brett character". Food Chem 114:15-19.

5. Albino EA. 2011. A survey of Brettanomyces/Dekkera strains for differences in aroma production. Thesis, University of California, Davis.

Joseph CML, Gorton LW, Ebeler SE and Bisson LF. 2013. Production of volatile compounds by wine strains of Brettanomyces bruxellensis grown in the presence of different precursor substrates. Am J Enol Vitic 64:231-240.

Joseph CML, Albino EA, Ebeler SE and Bisson LF. 2015. Brettanomyces bruxellensis aroma-active compounds determined by SPME GC-MS olfactory analysis. Am J Enol Vitic 66:379-387.
6. Conterno L, Joseph CML, Arvik TJ, Henick-Kling T and Bisson LF. 2006. Genetic and physiological characterization of Brettanomyces bruxellensis strains isolated from wines. Am J Enol Vitic 57:139-147.

7. Chatonnet P, Dubourdieu D, Boidon JN and Pons M. 1992. The origin of ethyl-phenols in wine. J Sci Food Agric 60:165-178.

Loureiro V and Malfeito-Ferreira M. 2003. Spoilage yeasts in the wine industry. Int J Food Microbiol 86:23-50.

8. WLD medium is Wallerstein Laboratory Differential medium and is commercially available. This medium allows selection against $S$. cerevisiae because it contains the antibiotic cycloheximide. Brettanomyces is resistant to cycloheximide and will grow on this medium displaying a distinctive colony morphology. MRS (de Man, Rogosa and Sharp) medium was designed to enrich for growth of the lactic acid bacteria and is used to detect these organisms in wine.

9. Heretszyn T. 1986. Formation of substituted tetrahydropyridines by species of Brettanomyces and Lactobacillus isolated from mousy wines. Am J Enol Vitic 37:127-132. 